\title{
Pompéi. L'atelier de potier de la via dei Sepolcri, 29
}

Campagne 2013

Laetitia Cavassa, Bastien Lemaire, Guilhem Chapelin, Aline Lacombe, John-Marc Piffeteau et Giuseppina Stelo

\section{(2) OpenEdition \\ 12 Journals}

Édition électronique

URL : http://journals.openedition.org/cefr/1139

DOI : $10.4000 /$ cefr. 1139

ISSN : 2282-5703

Éditeur

École française de Rome

Référence électronique

Laetitia Cavassa, Bastien Lemaire, Guilhem Chapelin, Aline Lacombe, John-Marc Piffeteau et Giuseppina Stelo, "Pompéi. L'atelier de potier de la via dei Sepolcri, 29 », Chronique des activités archéologiques de l'École française de Rome [En ligne], Les cités vésuviennes, mis en ligne le 06 mars 2014, consulté le 11 septembre 2019. URL : http://journals.openedition.org/cefr/1139; DOI : 10.4000/ cefr. 1139

Ce document a été généré automatiquement le 11 septembre 2019

(c) École française de Rome 


\title{
Pompéi. L'atelier de potier de la via dei Sepolcri, 29
}

\author{
Campagne 2013
}

\begin{abstract}
Laetitia Cavassa, Bastien Lemaire, Guilhem Chapelin, Aline Lacombe, John-Marc Piffeteau et Giuseppina Stelo
\end{abstract}

\section{NOTE DE L'AUTEUR}

Cette opération fait partie du projet « Organisation, gestion et transformations d'une zone suburbaine : le secteur de la Porte d'Herculanum à Pompéi, entre espace funéraire et commercial » porté par Laetitia Cavassa (Centre Jean Bérard), Nicolas Laubry (Université de Paris Est-Créteil-Val de Marne) et Nicolas Monteix (Université de Rouen). Ce projet s'insère dans le programme quinquennal (2012-2016) de l'École française de Rome et a obtenu une concession de fouilles pluriannuelle (2012-2016) accordée par le Ministero per $i$ Beni e le attività culturali.

L'équipe de fouille était composée de Guilhem Chapelin, architecte (Centre Jean Bérard), Léo Cagnard (doctorant à l'Université Paul-Valéry Montpellier III - UMR 5140), Saverio De Rosa, numismate, Giovanni Festa (étudiant à l'Università degli studi di Salerno) Aline Lacombe (céramologue, Direction Archéologie de la ville d'Aix-en-Provence), et JohnMarc Piffeteau (doctorant à l'Université de Nanterre - Paris X- EPHE, UMR 8210 ANHIMA). Ces recherches ont été poursuivies grâce aux crédits du Ministère des Affaires étrangères. Nous remercions la Soprintendenza speciale per i Beni archeologici di Napoli e Pompei pour nous avoir permis de travailler dans les meilleures conditions, la Dott.ssa

T. E. Cinquantaquattro, Soprintendente Speciale dei Beni Archeologici di Napoli e Pompei, la Dott.ssa G. Stefani, Directrice du site de Pompéi, le Dott. F. Galeandro, fonctionnaire responsable de notre secteur et V. Sabini, assistant scientifique.

1 La première opération de fouilles de l'atelier de potier de la via dei Sepolcri, 29 (fig. 1), durant laquelle les niveaux de 79 ap. J.-C. ont été dégagés, a eu lieu en 2012 . Une première étude avait alors été réalisée nous permettant d'établir le plan de l'ensemble de la 
structure et d'en identifier la production, avec la mise au jour de gobelets en paroi fine crus en phase de séchage lors de l'éruption ${ }^{1}$.

Fig. 1 - Pompéi. Plan des boutiques situées le long de la via dei Sepolcri.

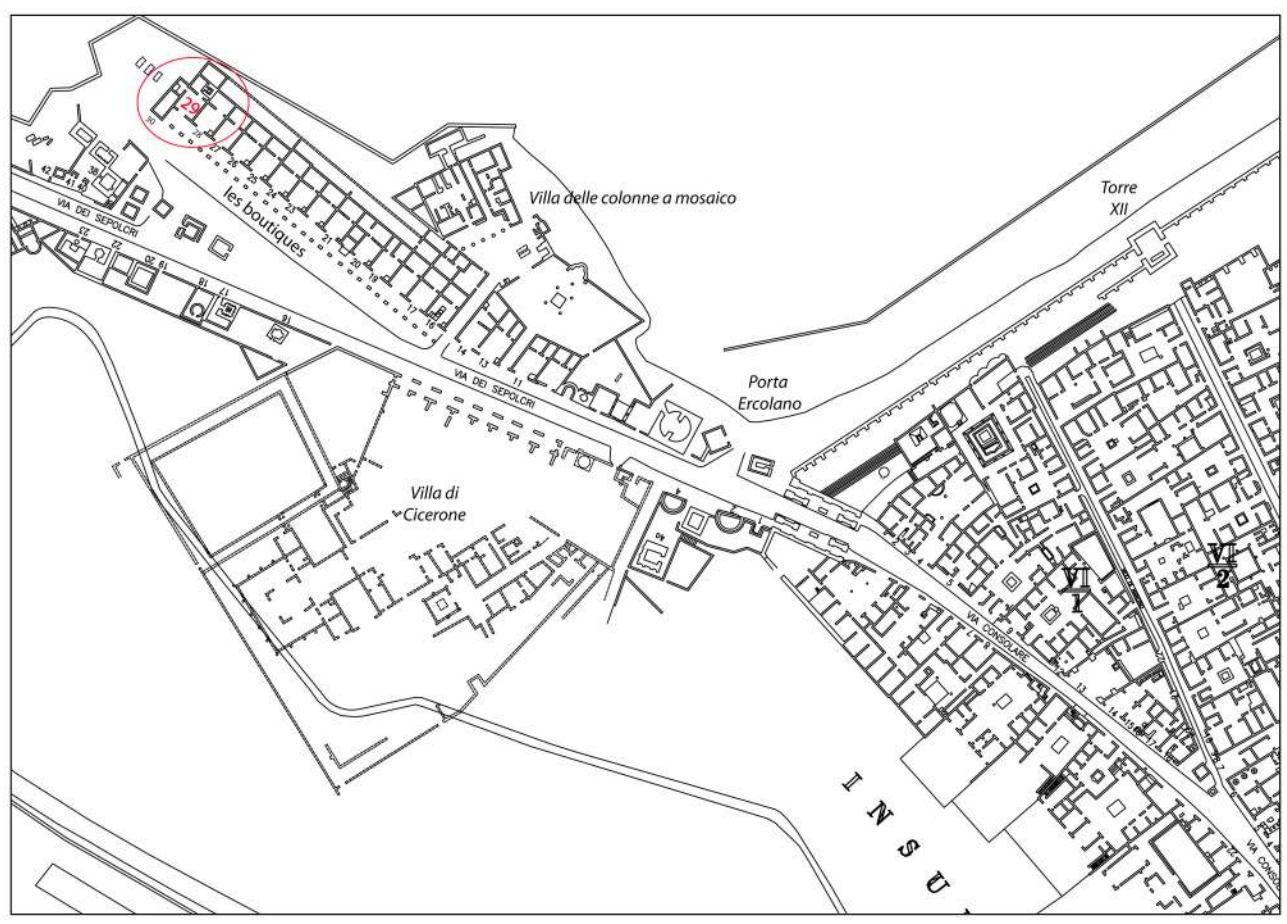

D'après A. Van der Poel, Corpus Topographicum Pompeianum : The Rica maps of Pompeii, 1983.

2 La campagne 2013, qui s'est déroulée du 9 au 27 septembre, nous a permis de définir les phases d'occupation de la boutique. Outre l'identification des différentes productions de cet atelier, les nombreux éléments découverts nous permettent déjà de dresser une chronologie des événements et des remaniements qui ont affecté la boutique et le portique construit en façade, depuis leur création jusqu'à l'éruption de 79 ap. J.-C.

3 En plus des pièces 1 et 2 partiellement fouillées l'année dernière, un nouvel espace (sondage 6) a été ouvert lors de cette campagne (fig. 2). 
Fig. 2 - Pompéi. Plan de la boutique n. 29.

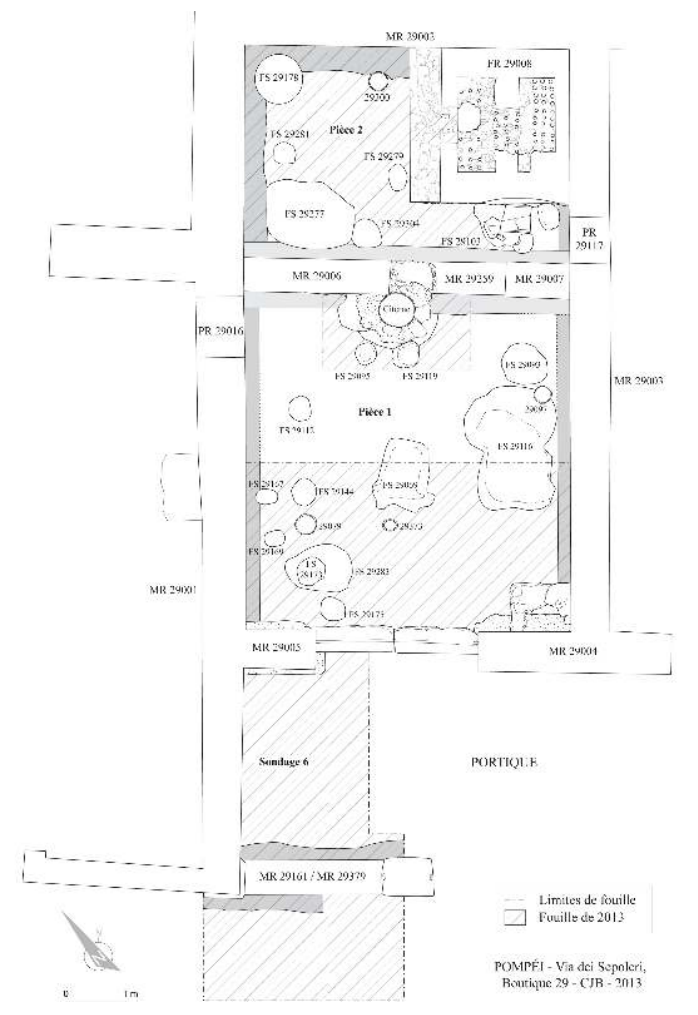

G. CHAPELIN, B. LEMAIRE.

4 Ce sondage se situe devant la boutique 29, le long du mur MR29001, dans l'emprise du portique. Il se présente sous la forme d'un rectangle mesurant $5,20 \mathrm{~m}$ du nord au sud et $3,10 \mathrm{~m}$ d'est en ouest, aménageant une surface d'environ $16 \mathrm{~m} 2$. Il offrait la possibilité d'observer l'espace de circulation du portique, en avant de la boutique, ainsi que les niveaux qui font le lien entre le portique et la voie plus au sud, la via superior. Ce sondage a apporté des informations sur la construction du portique et ses remaniements mais, il n'a pas été possible de l'étendre jusqu'à la voie pour mettre en évidence les éléments qui palliaient le dénivelé et font le lien entre la voie et le portique (escalier, rampe).

\section{État IA : les premiers niveaux de fréquentation}

5 L'état le plus ancien, partiellement observé en 2012, a été identifié sur l'ensemble de la boutique (pièces 1 et 2) et probablement au fond du sondage du portique. Il s'agit de deux couches sur lesquelles la boutique et le portique ont été fondés.

6 Le plus ancien niveau identifié, probablement géologique, est une épaisse couche argileuse jaune (US 29071). Il a été repéré de l'extrémité nord de la boutique (pièce 2), jusqu'au niveau de l'entrée (pièce 1) et très probablement dans la partie sud du sondage 6 entre la voie et le portique (US 29405). Cette couche présente une nette rupture et plonge à partir du tiers sud de la pièce 1.

7 Afin de combler cette dépression, un niveau de limon sableux noir (US 29072) semble être installé. Cette couche est composée de sable noir assez grossier et se poursuit plus au sud sous le seuil, servant probablement de remblai d'installation au niveau de circulation du 
portique. Il se pourrait que cet épais remblai soit retenu au sud par le mur de fondation des arcades du portique, MR29379, car il n'a pas été observé de l'autre côté de celui-ci. Il s'agira de vérifier cette hypothèse lors de la prochaine campagne de fouilles.

\section{État IB : l'installation de la boutique}

8 L'ensemble de la boutique semble directement installé dans les niveaux de l'état précédent. Il nous a été possible d'identifier et de fouiller tout ou partie des fondations des trois principaux murs de la boutique 29 (MR29001, MR29002 et MR29003).

9 Après l'installation des trois principaux murs de la boutique, le mur MR29259 est construit, venant s'adosser aux murs est et ouest et séparant deux espaces : les pièces 1 et 2. Sa tranchée de fondation vient recouper celles des deux autres murs (MR29001 et MR29003).

L'ensemble de ces murs a été fondé dans des tranchées assez profondes. Leur élévation repose sur une semelle de fondation en blocage, probablement damé, au vu de la texture très compacte du matériau employé. Le matériel mis au jour dans le remplissage des tranchées, bien que peu abondant, permet d'envisager la construction de la boutique

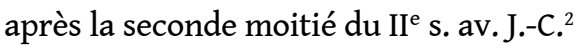

11 Les murs MR29004 et MR29005, formant l'entrée de la boutique, semblent bien postérieurs et appartiennent probablement à son dernier état.

12 La boutique et le portique appartenant à un seul programme de construction, les mêmes constatations sont faites pour le sondage 6 dans lequel les niveaux de fondation du mur MR29279, soutenant les arcades du portique et maintenant en place le niveau de circulation de ce dernier ont été atteints. Ce mur repose sur une semelle de fondation qui semble être du même type que celles observées pour les murs de la boutique. Le remplissage semble également être de même nature que pour les autres tranchées. Peu de matériel a été mis au jour. Entre autres un bord de bol à vernis noir donnerait un terminus post quem du III ${ }^{\mathrm{e}} \mathrm{-II}$ e s. av. J.-C.

\section{État IIA : les premiers aménagements}

13 Une fois les boutiques et le portique construits, une première utilisation des lieux remontant à un état antérieur à la construction du four et à l'existence de l'atelier de potier est attestée par l'installation de fosses.

14 Dans la première pièce, au sud-ouest du seuil, une fosse (FS29283) creusée dans un niveau de remblai est installée. Son comblement était composé de nombreux fragments de tegulae, d'une amphore punique de type Maña C2c, ainsi que d'un bord d'assiette en sigillée locale de type Conspectus 1.1 fournissant une datation dans la seconde moitié du I ${ }^{\text {er }}$ siècle av. J.-C. Il s'agit d'une des seules installations antérieures au sol bâti (SL29062) et aux grands remaniements qui auront lieu par la suite.

15 Dans la pièce 2, plusieurs fosses antérieures à l'installation du four ont été fouillées (FS29304, FS29277, FS29281, FS29178, FS29103), dont le remplissage, riche en matériel céramique, fournit une datation autour de la fin du $\mathrm{II}^{\mathrm{e}}$ siècle avant notre ère. 


\section{État IIB : la mise en place de l'atelier de potier}

16 Cet état correspond à un grand réaménagement de la boutique et peut-être du niveau de circulation du portique. En effet, c'est à cette époque que le four, la voûte maçonnée nord-sud (VT29020) soutenant le premier étage et la citerne semblent être installés.

17 L'installation du four et de la voûte dans la pièce 2, semblent appartenir à un seul programme de construction: la voûte a été spécialement aménagée de façon à laisser passer un conduit relativement large, permettant aux fumées générées par le four de s'échapper en traversant les pièces de l'étage jusqu'au système de couverture de l'édifice (fig. 3).

Fig. 3 - Pompéi. Photo du four et détail de son système d'évacuation des fumées.

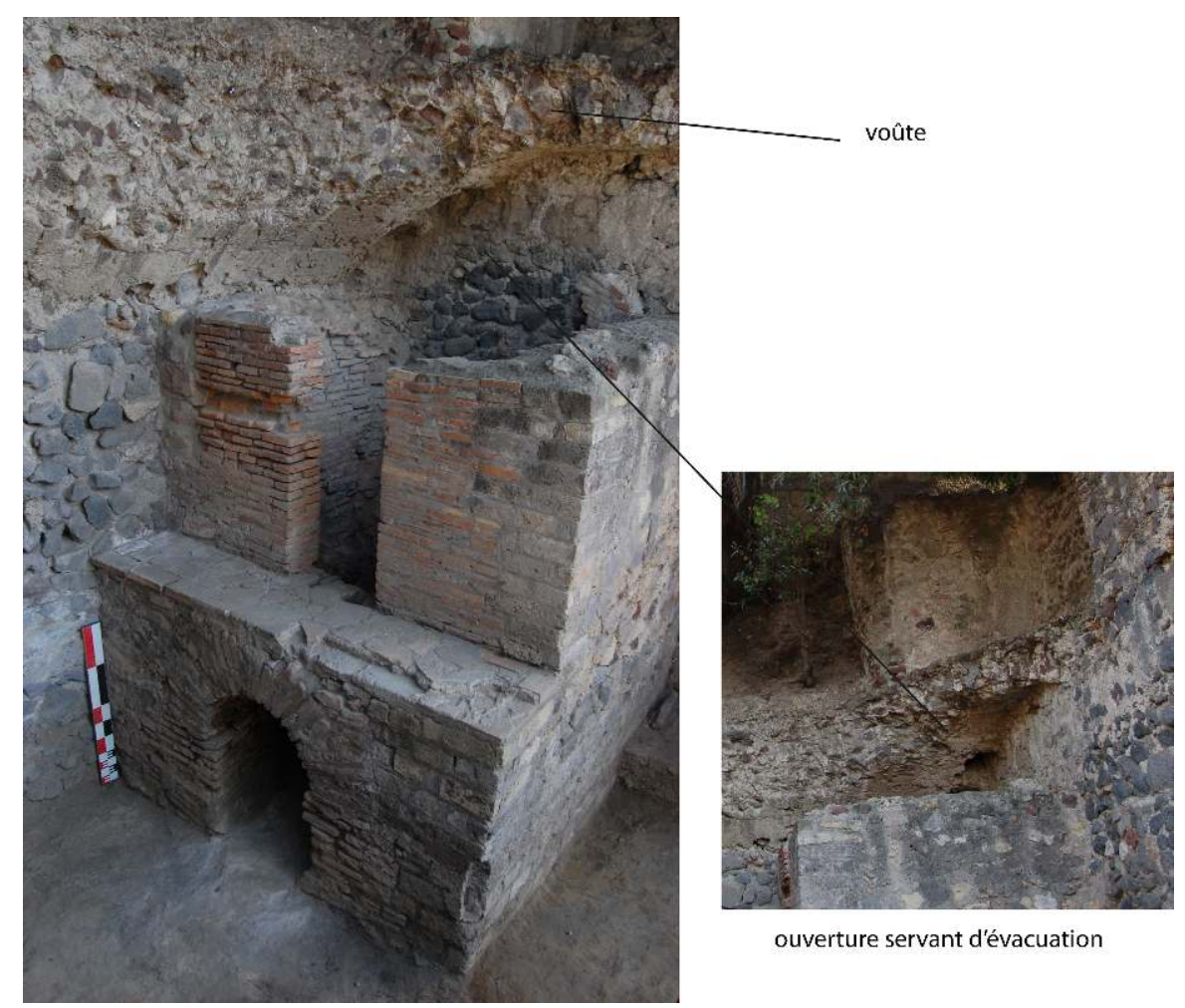

Centre Jean Bérard.

Le four est construit en blocs de tuf sur ses façades extérieures, à l'exception des passages qui mènent à la chambre de chauffe et au laboratoire, qui sont exclusivement réalisés en tegulae retaillées. L'intérieur du four est également composé de tegulae, dont les qualités réfractaires sont plus appropriées (fig. 4). Ses murs reposent sur des fondations coulées en mortier d'environ 20 à $25 \mathrm{~cm}$, remplissant des tranchées peu profondes qui recoupent les états antérieurs. 
Fig. 4 - Pompéi. Le four vu de l'ouest.

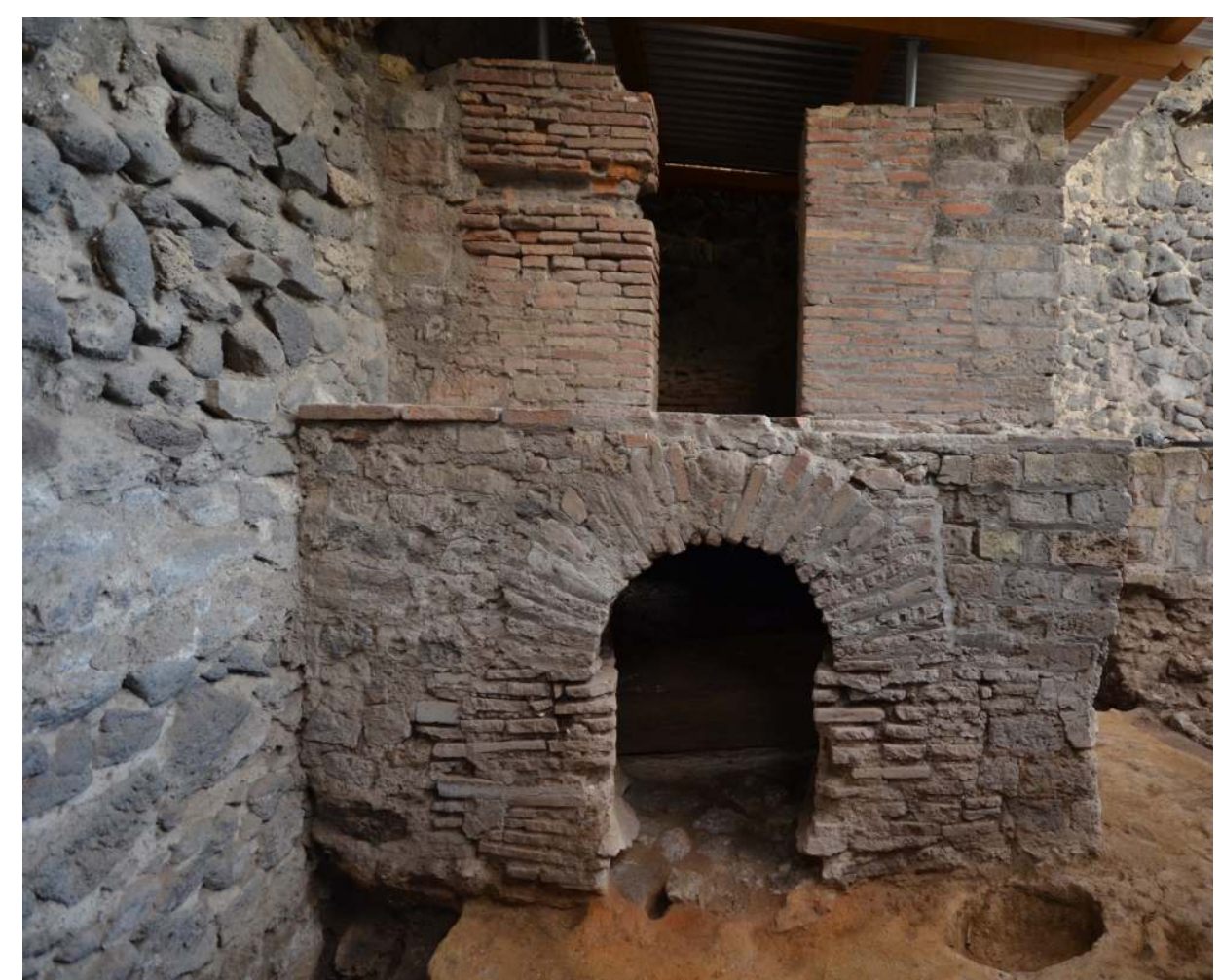

Centre Jean Bérard.

19 Pour des raisons techniques, la fouille de cette année n'a pas pu concerner l'ensemble des niveaux présents dans la chambre de chauffe. Cependant, il a été possible de fouiller l'alandier (0,85 sur 0,70 m), présentant les mêmes niveaux que ceux de la chambre de chauffe, ce qui a permis d'avoir une coupe stratigraphique de l'ensemble des niveaux de fonctionnement du four, depuis sa fondation jusqu'à son état final de 79 de notre ère.

Il s'agit du sol bâti (SL29367), mesurant $10 \mathrm{~cm}$ d'épaisseur et constitué de blocs de basalte et de blocs de remploi liés à la terre (fig. 5). 
Fig. 5 - Pompéi. Détail des niveaux de construction du four à l'intérieur de l'alandier.

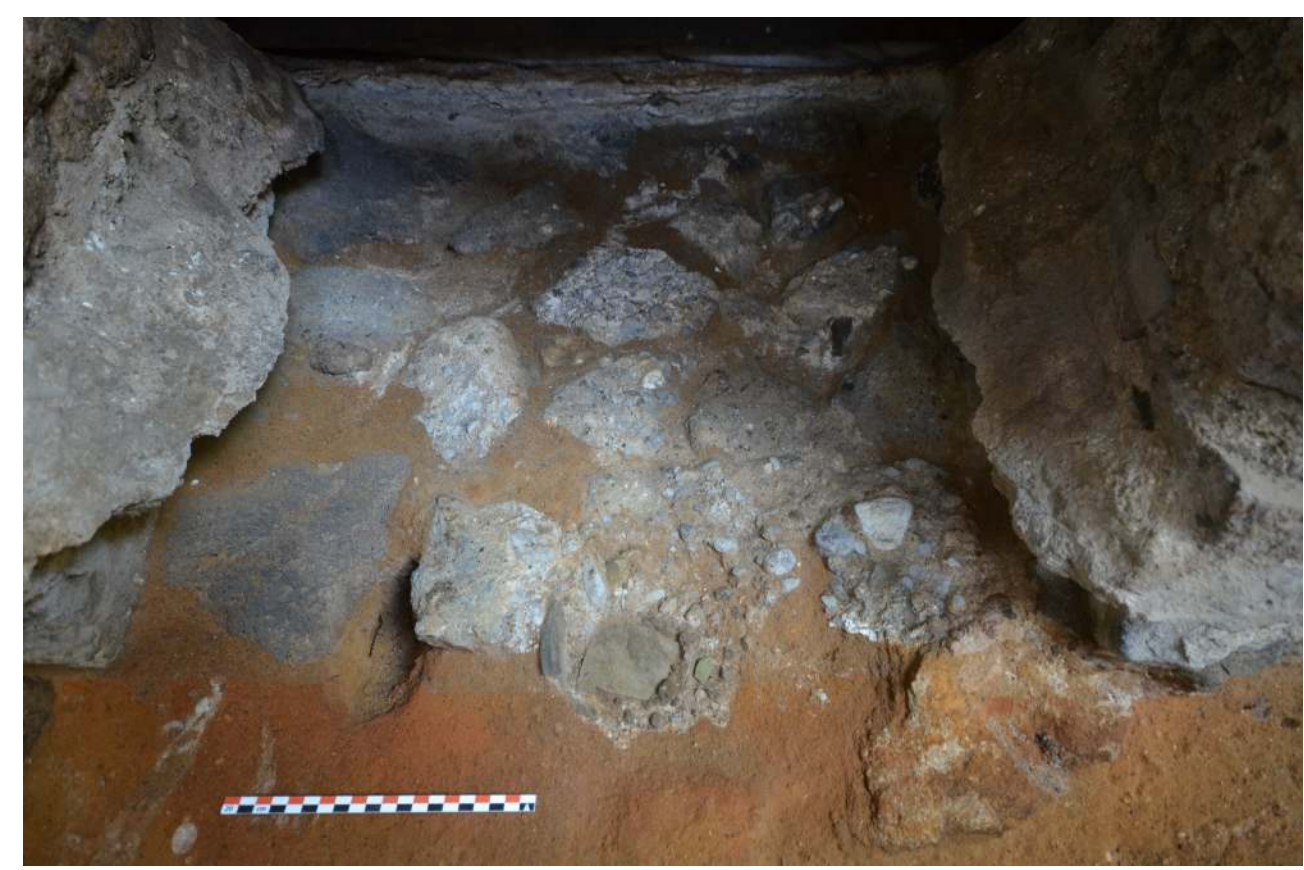

Centre Jean Bérard.

21 Ce sol s'enchâsse légèrement sous les murs du four, indiquant qu'il s'agit d'un seul programme de construction et non de plusieurs phases et réaménagements. Les blocs de remploi (8 à $10 \mathrm{~cm}$ d'épaisseur) sont des fragments d'un sol solidement bâti présentant plusieurs couches (des galets, un mortier grossier et une couche de mortier plus fin). Des éléments identiques ont été identifiés dans la voûte de la pièce, ce qui pourrait être un indice supplémentaire de la contemporanéité de leur construction.

Dans le reste de la pièce, un niveau de circulation (SL29135), en connexion avec le sol de l'alandier (SL29367) recouvrant les fosses de l'état précédent est installé. Son caractère très charbonneux correspond très probablement à l'utilisation du four.

L'installation de la citerne dans la pièce 1, entraîne la condamnation du passage entre les deux pièces qui se trouve par conséquent décalé vers l'est. Un sol bâti (SL29062) est également mis en place. Ce dernier est creusé d'un nombre important de fosses ${ }^{3}$, dont certaines sont clairement associées à l'installation d'amphores, « fichées » dans le sol. La fonction de ces amphores comme éléments de stockage (ou autres) n'est pas bien identifiée car elles ont souvent été détruites et parfois mélangées à d'autres déchets, afin de combler les fosses dans un état postérieur (état III).

\section{État IIIA : un réaménagement}

24 Cet état est caractérisé par la condamnation de la citerne et le comblement des amphores du précédent état.

Suite à une dégradation progressive ou à un événement plus important comme un tremblement de terre, une partie de la citerne s'effondre. Une brèche relativement importante se forme à l'ouest du mur de la citerne, fragilisant cette dernière et détériorant probablement la qualité de l'eau stockée. Elle est ensuite bouchée dans sa 
partie supérieure au niveau des assises maçonnées, par la mise en place de trois gros fragments de panse d'amphore italique (fig. 6).

Fig. 6 - Pompéi. La citerne et le bouchage de la brèche.

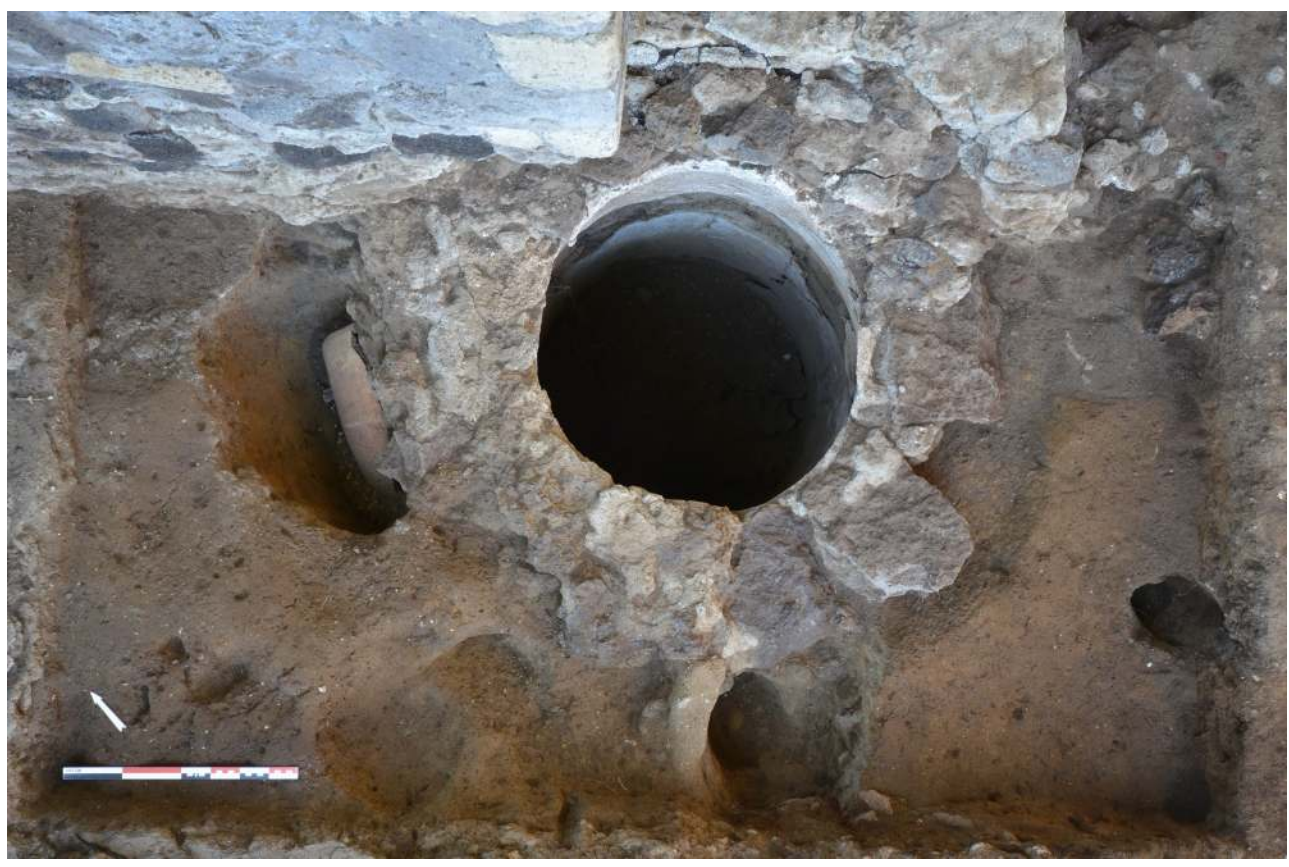

Centre Jean Bérard.

26 Les fosses observées dans l'état précédent, fonctionnant avec le sol SL29062, sont comblées lors de cet état ou durant le suivant (état IIIB), correspondant à la création d'un dernier niveau de sol.

Le comblement de ces fosses a restitué une quantité de matériel très intéressant, et plus particulièrement des fragments de céramique à paroi fine surcuits témoins de la production.

Ces données sont tout à fait déterminantes car elles permettent de reconstituer en partie l'histoire de l'atelier. En l'absence de données chronologiques précises sur la construction même du four, la présence de ces surcuits permet d'établir un terminus post quem. Il s'agit pour l'essentiel de gobelets à décors sablés dont la production est attestée sous Tibère. Ces gobelets sont surcuits et ont été jetés entiers dans ces fosses (fig. 7 et 8). 
Fig. 7 - Pompéi. Gobelets surcuits, en céramique à paroi fine mis au jour dans le remplissage des fosses de la pièce 1 .
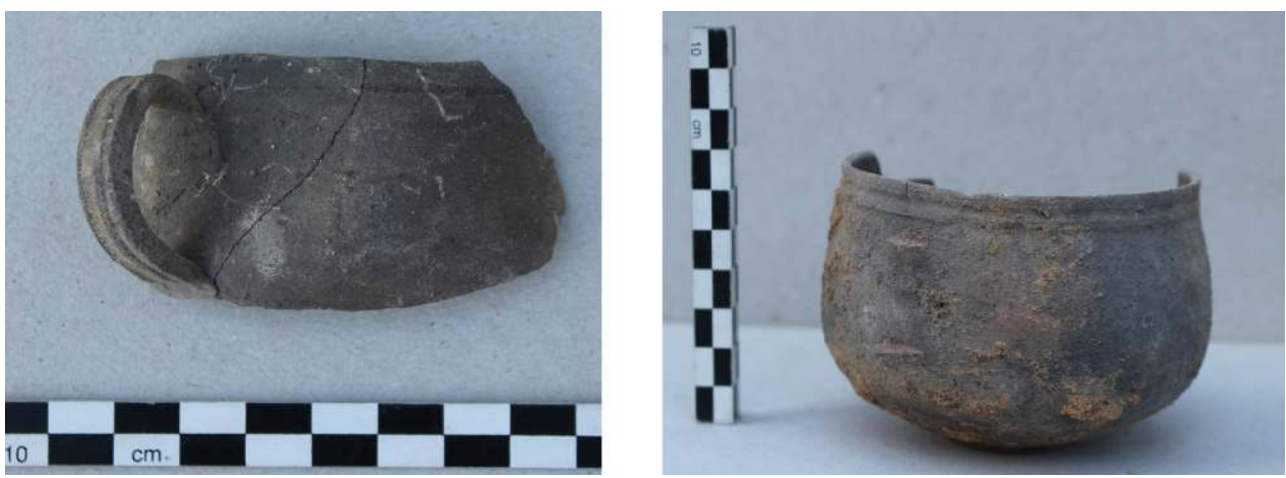

Centre Jean Bérard.

Fig. 8 - Pompéi. Gobelets en céramique à paroi fine, à décor sablé, mis au jour dans le remplissage des fosses de la pièce 1 .

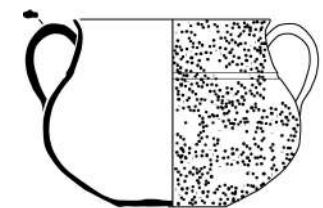

29174-06

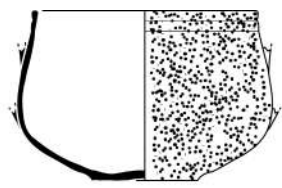

29174-08

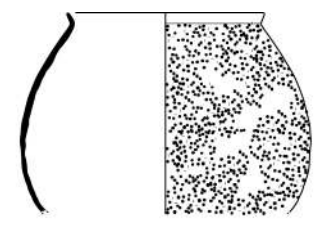

29174-07

Dessins A. Lacombe

\section{État IIIB : les niveaux de 79 ap. J.-C.}

Ce dernier état se caractérise par le recouvrement des fosses de l'état précédent et de la citerne, dans le but d'installer un dernier niveau de sol dont il ne nous reste que les éléments présents dans la partie sud-ouest de la pièce 1. La conservation des couches de remblais de nivellement et du niveau de sol associé s'explique par l'existence de la structure bâtie SB29033, fouillée l'année passée. Cette structure, principalement réalisée en fragments de tegulae et fonctionnant avec l'amphore 29079, semble correspondre au dernier sol installé dans la boutique (SL29401).

La fouille de cette année a notamment permis de réviser un point important. En effet, dans l'angle sud-est de la pièce 1, le niveau 29120 n'est pas un reliquat du dernier sol de la boutique, mais la semelle de fondation du mur MR29004. Un niveau identique (US 29182) se trouve de l'autre côté du seuil, servant de semelle de fondation au mur de façade de la boutique, MR29005. L'ensemble des semelles de fondation présente une technique de construction uniforme avec un lissage de leur surface au mortier.

Le dernier sol correspondant au niveau de 79 ap.J.-C., a donc totalement disparu, probablement depuis son dégagement lors des fouilles des siècles antérieurs et son érosion à l'air libre. Seule la structure en tuiles SB29033 permet d'observer ce niveau, ainsi que les deux semelles de fondations précédemment décrites. En effet, ces fondations 
coulées sont plus élevées que le sol SL29062 de l'état II, prouvant bien qu'elles ont été réalisées dans un sol plus haut, aujourd'hui disparu.

À ce dernier état, appartient également la base de l'escalier (BS29059) située dans l'angle sud-est de la pièce 1 permettant d'accéder au premier étage, car elle repose sur la semelle de fondation du mur MR29004 (US 29120). Cependant, même si cette structure n'a pas été démontée pour des raisons de conservation, il est possible que les niveaux observés sous cette base lui servant de fondation, aient également pu appartenir à une base d'escalier d'un état antérieur.

Le même phénomène semble s'être produit dans l'espace de circulation du portique. En effet, le sol observé (SL29177) correspond probablement au pendant du sol SL29062 de la pièce 1 et se trouve lui aussi plus bas que les fondations coulées des murs MR29004 et MR29005. Cela prouve bien que les fondations de ces murs ont été creusées dans un niveau aujourd'hui disparu. De plus, il est à noter que ces murs sont les plus tardifs de la boutique, comme l'attestent leur fondation, ainsi que leur construction en blocs de tuf et en tegulae retaillées. Le remaniement de ces murs de fermeture se retrouve dans les autres boutiques du portique et semble donc correspondre à un programme de réaménagements tardifs de cet ensemble architectural.

Le dernier état du sol de la pièce 2 (SL29031) a été mis en évidence l'année dernière. Il présentait une texture compacte malgré son aspect très cendreux, probablement en raison des cendres issues du four. C'est sur ce dernier niveau que reposaient les vases crus, prêts à être cuits dans le four, retrouvés dans l'angle sud-ouest de la pièce sous une fine couche de lapilli encore intacte, provenant de l'éruption de 79.

\section{Conclusion}

La campagne de fouille de septembre 2013 a apporté des premiers éléments de datation concernant la fondation de la boutique 29 et donc de l'ensemble du portique et de ses remaniements postérieurs. Elle a aussi permis d'encadrer chronologiquement le fonctionnement et la production de l'atelier de potier de la Porta Ercolano. En l'état actuel de l'étude du matériel céramique, il semble probable que l'atelier fonctionnait depuis l'époque de Tibère, voire un peu avant. Quant à la production, seuls les vases en céramique à paroi fine sont pour le moment clairement attestés comme ayant été produits dans l'atelier.

\section{NOTES}

1. Voir Chronique des activités archéologiques de l'École française de Rome [En ligne], URL: http:// cefr.revues.org/881.

2. L'étude du matériel étant en cours, toutes les datations fournies dans l'article sont préliminaires.

3. Cinq fosses ont été mises au jour au total : FS29112, FS29173, FS29144, FS29095 et FS29119. Les deux dernières ont été fouillées durant la campagne de 2012. 


\section{INDEX}

\section{Index géographique : Pompéi}

Mots-clés : four de potier, gobelets à paroi fine, céramique, urbanisme, portique institutions Centre Jean Bérard (USR 3133 CNRS-EFR), Soprintendenza speciale per i Beni archeologici di Napoli e Pompei, Ministère des affaires étrangères (Paris)

\section{AUTEURS}

\section{LAETITIA CAVASSA}

Centre Jean Bérard (USR 3133 CNRS-EFR) - laetitia.cavassa[at]cnrs.fr

\section{BASTIEN LEMAIRE}

Université Paul-Valéry Montpellier III, UMR 5140 Archéologie des Sociétés Méditerranéennes (CNRS-UPV) - bastien_lemaire[at]hotmail.fr

\section{GUILHEM CHAPELIN}

Centre Jean Bérard (USR 3133 CNRS-EFR)

\section{ALINE LACOMBE}

Direction Archéologie Aix-en-Provence

JOHN-MARC PIFFETEAU

Université de Nanterre - Paris X- EPHE, UMR 8210 ANHIMA

\section{GIUSEPPINA STELO}

Centre Jean Bérard (USR 3133 CNRS-EFR) 